\title{
Diseño de un modelo de articulación entre la cadena productiva hortofruticola colombiana y la cadena logística de frio
}

\section{Design of a model of interaction between the colombian fruit\&vegetal productive chain and logistics cold chain}

\author{
Diego Hernando Flórez Martínez ${ }^{1}$ \\ Recibido para publicación: Junio 24 de 2017 - Aceptado para publicación: Noviembre 23 de 2017
}

\begin{abstract}
RESUMEN
El objetivo de esta investigación es proponer un modelo de articulación, entre la cadena productiva (CP) hortofrutícola colombiana como mecanismo de gestión de las interacciones entre actores para el flujo de materiales y la cadena de frío (CF) como herramienta logístico-tecnológica, para la conservación de alimentos perecederos. A partir de una metodología secuencial de construcción, se propone un modelo de eslabones y segmentos para el sector hortofrutícola colombiano, que integra las actividades desde los proveedores de insumos hasta el cliente o consumidor final, identificando sus descriptores. Posteriormente se analizan los modelos de cadena de frío propuestos en la literatura conceptualización e identificación de puntos críticos de control tanto exógenos como endógenos con base en el sistema de análisis de riesgos y puntos críticos de control (HACCP), seleccionando el modelo de cadena sincronizada. El análisis conjunto y superpuesto de estos dos modelos, se convierte en el diseño articulado, que permite direccionar la adaptación de tecnología de transporte y almacenamiento de productos bajo condiciones de temperatura y humedad determinadas dentro de la cadena productiva hortofrutícola, como factor de ventaja competitiva.
\end{abstract}

Palabras clave: Cadena productiva, cadena de frío, cadena de suministros, calidad de alimentos, hortofrutícola

\begin{abstract}
The objective of this research is to propose a model to articulate Colombian horticultural production chain as a mechanism for managing the interactions between actors for the flow of materials with the cold chain as a logistictechnological tool for the conservation of perishable foods. Based on a sequential construction methodology, a link and segment model is proposed for the Colombian horticultural sector. The model integrates the activities from the input suppliers to the final customer or consumer, identifying their descriptors. Consequently, the cold chain models proposed in the literature are conceptualized and characterized trough a critical control point's analysis (exogenous and endogenous), based on the HACCP system, selecting the synchronized supply chain model. The joint and superimposed analysis of these two models becomes the articulated design, which allows directing the adaptation of technology in transportation and storage of products under appropriated cold chain conditions for fruits and vegetal products as a competitive advantage.
\end{abstract}

Key words: Productive chain, cold chain, horticulture, supply chain, food quality.

\footnotetext{
${ }^{1 *}$ MSc. Profesional de planeación estratégica en la Corporación Colombiana de Investigación Agropecuaria - Corpoica. dhflorezm@ bt.unal.edu.co.
} 


\section{INTRODUCCIÓN}

Según la organización de las naciones unidas para la agricultura (por sus siglas en ingles FAO), a nivel mundial la producción de frutas como la de hortalizas han experimentado crecimientos sostenidos durante los últimos años; igualmente el valor la producción frutícola se ha incrementado desde 1990 a una tasa del 10,7\% anual (MADR, 2016). El comportamiento global de frutales y hortalizas, se caracteriza por las tendencias particulares de los diferentes nichos de mercado donde desde una perspectiva macro regional, a nivel continental Asia con el $74 \%$ de la producción es el principal proveedor de estos productos, le siguen en importancia Europa y América Latina y el Caribe con el $10 \%$ y $9 \%$ respectivamente (MADR, 2016).

Estos nichos comparten un factor común en que los productos de la cadena hortofrutícola, requieren de un manejo sistémico de variables organolépticas tales como el sabor, color, humedad, contenido de azúcares entre otras las cuales se deben mantener hasta llegar al cliente final; ya que son afectadas por factores exógenos como temperatura, presión, carga biológica y carga química del entorno. El éxito de este proceso recae en un manejo efectivo de la "CF", término usado para describir la serie de operaciones interdependientes en la producción, distribución, almacenamiento y comercialización de productos congelados a través los procedimientos y equipos para mantener la temperatura deseada (Torres, 2006).

Lo anterior debido a que, a nivel mundial, el manejo de las actividades en cosecha, poscosecha y transformación de frutas $y$ hortalizas, se ve fuertemente influenciado por la implementación de insumos tecnológicos y no tecnológicos, con el objetivo de incrementar la vida útil de los productos (Montanari 2008), y así disminuir los volúmenes de pérdidas que se presentan y que, para el caso colombiano, puede superar el $50 \%$ de la producción (Floréz y Cifuentes, 2013).

En Colombia el seguimiento a los productos hortofrutícolas abarcar tanto la producción de frutas frescas, vegetales y granos, como la transformación industrial en bienes como jugos, enlatados, mermeladas, compotas, pulpas y salsas. Es decir, se pueden diferenciar dos componentes clave articulados dentro del subsector. Estosson, el sector netamenteagrícola, que comprende las actividades de cosecha, sostenimiento, recolección y tratamiento primario del producto y el sector industrial que abarca la transformación y generación de valor agregado (Asohofrucol y MADR, 2012); posteriormente se desarrollan las actividades de comercialización de los productos.

Las nuevas condiciones de mercado hacen necesario mejorar la coordinación entre los productores, la industria y la distribución, a través de esquemas de interacción como las cadenas productivas (Castro y Lima, 2001). De acuerdo con Cuevas (2005), la CP es el conjunto de componentes que interactúan en el entorno del agronegocio de un país o región, que incluye a los sistemas productivos, proveedores de insumos y servicios, industrias de producción y transformación, agentes de distribución y comercialización y a los consumidores. El concepto de CP se desarrolló como herramienta de gestión, evaluación y diagnostico con un enfoque desde el análisis sistémico, donde los flujos de material, de capital y de información son los mecanismos de relación entre los diversos agentes involucrados (Castro et al., 1998), para la generación de valor agregado.

La tendencia actual en el comercio de productos alimenticios, se enfoca cada vez más hacia alimentos de alto valor agregado (Montanari, 2008); (Rodríguez et al., 2011), donde es de particular interés el poder integrar, no solo los procesos sino cada uno de los actores que conforman e integran los aparatos productivos, han evolucionado hacia un mayor 
énfasis en la calidad, concepto que abarca múltiples dimensiones de un producto (Garcia y Poole, 2004; Garcia, 2009); mejorar el acceso a recursos clave e incrementar la eficiencia del sistema (Fischer et al., 2007), generando una fuente fundamental de competitividad, que algunas veces se denomina "ventaja colaborativa" o "ventaja comparativa de la cooperación", donde la integración de actores en distintos niveles del flujo de productos, es necesaria para minimizar costos y optimizar operatividad y calidad (Fischer et al., 2008).

El factor de calidad en productos perecederos y en particular aquellos del sector hortícola, parte de la concepción de "calidad percibida" por el cliente frente a los productos y de la "calidad ofrecida" por el productor, en relación con el proceso de conservación al cual son sometidos bajo mecanismos de conservación específicos (Likar, 2006). La CF como mecanismo de conservación del producto en etapa de poscosecha, implica manejos diferenciados y criterios particulares, de la misma manera en que se contextualiza con el manejo de costos y la crisis energética (Jol, 2007).

La CF es entonces, un sistema de redes logísticas dentro de un aparato productivo, equipado con tecnología en transporte y almacenamiento que garantiza la calidad de productos frescos y congelados de carácter alimenticio, desde el productor hasta el consumidor (Wang, 2008). Esto en condiciones de baja temperatura que para el caso particular de productos frescos o refrigerados varía entre $0{ }^{\circ} \mathrm{C}$ y 10 ${ }^{\circ} \mathrm{C}$, mientras que para productos congelados debe ser inferior a los $0^{\circ} \mathrm{C}$ (Beilin 2004).

La investigación desarrollada en este documento busca orientar, a partir del conocimiento del desarrollo actual en $\mathrm{CF}$, el análisis metodológico de los modelos actuales de CF para cadenas logísticas, la articulación con el modelo de CP de eslabones y segmentos, que permiten relacionar de una manera clara de qué manera el producto llega del productor primario al cliente o consumidor final, a partir de los puntos críticos de control (Friend, 2001).

El objetivo de esta investigación es definir un modelo de integración específico a las particularidades de la cadena hortofrutícola colombiana, que, a través de la articulación de herramientas tecnológicas de cadena de frío, contribuya a mejorar la calidad e inocuidad de los productos, la trazabilidad a través de los canales de distribución y el monitoreo y seguimiento de variables como temperatura y humedad.

\section{MATERIALES Y METODOS}

El diseño metodológico de esta investigación considera cuatro etapas secuenciales que integran conceptos teóricos y herramientas procedimentales específicas, para diseñar el modelo de integración de la Cadena de frio (CF) a la Cadena productiva (CP).

\section{Etapa 1 - Diseño de la Cadena Productiva:}

El modelo de la CP hortofrutícola a diseñar, estructura las bases conceptuales y metodologías establecidas en los estudios de caracterización sectoriales realizados por el MADR en el marco de la Dirección de cadenas productivas, donde toman como referencia los modelos previamente considerados, estructurados a través de procesos de investigación de diferentes entidades que apoyan y soportan al sector en el país, lo que permite abordar de manera global la interacción de los actores a través de todo el proceso productivo (Castellanos et al., 2010).

Para diseñar la CP se implementó la metodología diseñada por Castro y Colaboradores (1998); adaptada para el caso colombiano (Castro, 2006). En la tabla 1, se sintetizan los elementos de construcción utilizados.

De acuerdo con Cuevas (2005), los diferentes segmentos que se identifican en cada uno 
Tabla 1. Metodología de cadenas productivas agroindustriales.

\begin{tabular}{|c|c|}
\hline HERRAMIENTA & CONCEPTO \\
\hline $\begin{array}{l}\text { CADENA } \\
\text { PRODUCTIVA }\end{array}$ & $\begin{array}{l}\text { Conjunto de actores y unidades productivas que se relacionan para producir, } \\
\text { procesar, almacenar, distribuir y comercializar insumos y productos de origen } \\
\text { agropecuario o agroforestal. }\end{array}$ \\
\hline $\begin{array}{l}\text { FLUJOS DE MATERIAL } \\
\text { E INFORMACIÓN }\end{array}$ & $\begin{array}{l}\text { Conjunto de actores y unidades productivas que se relacio- } \\
\text { nan para producir, procesar, almacenar, distribuir y comerciali- } \\
\text { zar insumos y productos de origen agropecuario o agroforestal. }\end{array}$ \\
\hline ESLABONES & $\begin{array}{l}\text { Cada grupo de unidades productivas que se dedican a las actividades que } \\
\text { garantizan el desarrollo de los productos, entre las cuales existen diferencias } \\
\text { importantes en cuanto a sus tamaño, adopción tecnológica, naturaleza de la } \\
\text { propiedad entre otras variables que se pueden definir de segmentación, como } \\
\text { una analogía a la segmentación que se realiza en un mercado. }\end{array}$ \\
\hline SEGMENTO & $\begin{array}{l}\text { El concepto de segmento surge como un tipo de unidad productiva donde se } \\
\text { presenta de manera específica una o varias de las variables de segmentación } \\
\text { definidas. A partir del proceso de segmentación es posible entender la } \\
\text { composición de los eslabones de la cadena productiva y entender dinámicas } \\
\text { específicas de la cadena objeto de estudio, y de esta manera elaborar estrategias } \\
\text { diferenciadas de mejoramiento frente a necesidades identificadas para control } \\
\text { y seguimiento. }\end{array}$ \\
\hline $\begin{array}{l}\text { AMBIENTES } \\
\text { TRANSVERSALES }\end{array}$ & $\begin{array}{llllll}\text { Entendidos } & \text { como el conjunto de } & \text { estamentos, } & \text { instituciones, } \\
\text { políticas y normatividades que regulan a la cadena productiva, no } \\
\text { intervienen de manera directa en los flujos de material e información. }\end{array}$ \\
\hline
\end{tabular}

de los eslabones de la $\mathrm{CP}$, se relacionan con otros segmentos $\mathrm{u}$ otros eslabones a partir de flujos de recursos. Se pude determinar que desde el eslabón de proveedores hasta el consumidor final fluyen principalmente materia (materias primas, insumos, productos intermedios, productos finales), energía e información, y en la dirección contraria el flujo fundamental es de información y recurso financieros. Desde un punto de vista comercial se establece que el consumidor es quien dinamiza el desempeño de la CP.

\section{Etapa 2 -Selección del diseño de Cadena de Frío:}

El proceso de enfriamiento es un método clave para preservar sabor, textura y valores nutricionales de las comidas. Sin embargo, la eficacia, efectividad y eficiencia del mismo dependen de la selección de materiales, tratamientos previos, procesos de congelación, empaque, condiciones de almacenamiento, sistema de control y monitoreo (Cruz, 2009).

Los principales factores que inciden el diseño de la CF son el control de temperatura que varía en rangos desde $0^{\circ} \mathrm{C}$ hasta $-18^{\circ} \mathrm{C}$ (Wang, 2008); mecanismos de logística de distribución de alimentos perecederos, en condiciones más favorables para lograr una mayor frescura y calidad (Viteri, 2003), basados en mecanismos de enfriamiento $y$ congelación (en almacenamiento y transporte), para la conservación de las características organolépticas, control del abuso de la temperatura, gestióndelospuntos detransferencia y diseño de acciones preventivas (Fuller 1998).

La operación global dentro de la CF, es el enfriamiento o la congelación, el cual implica reducir temperaturas de los alimentos por debajo de la temperatura ambiente, pero por encima de 
$-1^{\circ} \mathrm{C}$, conel objetivodepromoverlaconservación eficaz a corto plazo de las características organoléptica, fisiológicas y nutricionales de los alimentos, retardando daños ocasionados por factores microbiológicos, físicos, así como reacciones químicas y bioquímicas asociadas con deterioro de los alimentos.

Sin embargo, esto es depende de los tiempos de comercialización y el tipo de productos, ya que los productos frescos son normalmente expuestos a temperaturas de $8-12{ }^{\circ} \mathrm{C}$, según el manejo y distribución logística establecida durante toda la cadena de suministros (Rediers ,2009), lo que contribuye a tener un nivel óptimo de trazabilidad del producto, así como el cumplimiento de limites estandarizados.

La implementación de esta logística implica una articulación directa con la producción primaria que asegure un abastecimiento continuo en cantidad y calidad a lo largo del año, teniendo en cuanta el flujo de material a través de la CF integrada a la cadena productiva buscando una eficiencia en el flujo económico sostenible, es decir garantizar en cada momento dentro del flujo de material asociado a los eslabones de la cadena, las condiciones de los productos con un rango de tolerancia establecido para las variables en seguimiento, donde para el caso particular de la cadena hortofrutícola, las condiciones de temperatura y humedad son prioritarias.

Se evalúan los modelos propuestos en la literatura sobre CF contemplando los siguientes factores:

- Tecnología asociada

- Gestión de las variables tiempo, temperatura y tolerancia (TTT)

- Elementos de asociación frente a la CP y/o cadena de suministros.

\section{Etapa 3 - elementos de interacción}

La herramienta de seguimiento básica para la CF y punto de partida para la concepción de una metodología de evaluación con connotaciones económicas, es un plan de temperatura (Cruz, 2009), el cual considera los eslabones desde la unidad de procesamiento, carga al transporte, el periodo de trasporte, a cada uno de los centros de distribución, la carga en el trasporte de distribución, el tiempo de transporte desde los centros de distribución, los puntos de comercialización, el ultimo comprador y el consumidor final. En este punto es más que evidente la necesidad de una "cadena integral de alimento" (Kuo, 2010), donde el manejo de la sensibilidad a la temperatura en productos perecederos, se debe mantener en una constante implementación y eficiencia en los métodos de transporte, almacenamiento, tecnologías de la información en la CF.

Se consideran como esquemas referentes para la integración de la CF a la CP los cuatro arquetipos de cadena de suministros de Forrester* (Cannella, 2010), usados para caracterizar los modelos de CF. El arquetipo de cadena sincronizada es el ideal para la integración.

- Tradicional: La regulación de la información y del flujo de material está dada por el proveedor, donde este controla el pedido del minorista teniendo en cuenta que no conoce el volumen real que necesita el cliente final. - Información compartida: Es este modelo el flujo de información dentro de la cadena de suministros, parte desde el cliente final, donde las cantidades solicitadas son conocidas tanto por productores, transformadores y comercializadores - Gestionada por el proveedor: integración de eslabones de manera horizontal, se presenta en los segmentos, donde algunos productores se articulan con transformadores y/o comercializadores para tener un inventario 
unificado en términos de la demanda del cliente final, esto limita la heterogeneidad de calidad del producto, pero disminuye la cobertura de actores dado que no todos manejan niveles tecnológicos similares. - Cadena sincronizada: pedidos se de manera puntual en la articulación de algunos segmentos de eslabón, lo cuales generan una cadena colaborativa dentro del modelo. En este caso existe comunicación directa entre los comercializadores y los transformadores para el manejo de las cantidades de los productores, así como el control en tiempo real del flujo de material.

\section{Etapa 4 - Diseño Esquema integrado del modelo:}

Contemplados los cuatro arquetipos de la cadena de suministros, que se presentan en cualquier actividad de logística y todos extensivos a la CP, el arquetipo ideal en función de las particularidades de este, es el de cadena sincronizada teniendo en cuenta la homogeneidad de información que se requiere en función de la temperatura del producto.

Sin embargo, los cuatro modelos pueden ser aplicados a cadenas puntuales de ciertos productos, considerando que este modelo ideal no contempla limitaciones como la localización geográfica de los miembros de la cadena, el tipo de demanda entendida para productos hortofrutícolas como la estacionalidad de la misma, las características del producto donde se tiene una heterogeneidad total entre productos en fresco, mínimamente procesados y de alto valor agregado, costos del sistema de trazabilidad (Ej. Sistemas de georreferenciación, Tecnologías de identificación de radio frecuencia, monitoreo en tiempo real, etc.), costos de insumos tecnológicos (inventario y transporte refrigerado), cambio organizacional en la consolidación de agronegocios sostenibles y grado de apertura de la información como una ventaja competitiva.
A continuación, se describen los resultados obtenidos en cada etapa a través del diseño metodológico secuencial.

\section{RESULTADOS Y DISCUSIÓN}

\section{Etapa 1:}

Los productos generados por el sector hortofrutícola son la base para estructurar la CP o cadena de valor, a partir del criterio de procesamiento y valor agregado de los mismos. La segmentación propuesta, a partir de la articulación entre eslabones, establece lineamientos de estructuración replicables en diferentes agronegocios y cadenas productivas alrededor de un producto específico. Esto concreta un modelo estándar de CP adaptativo y sistémico para los diferentes agronegocios. En la tabla 2, se establecen los eslabones y segmentos propuestos para la CP hortofrutícola.o.

A partir de la estructuración básica del sector hortofrutícola, se construye un modelo adaptativo para la CP hortofrutícola en el cual pueden identificarse los flujos de material y los flujos de capital entre las diferentes interrelaciones y articulaciones de la cadena. Sin embargo, teniendo en cuenta el impacto que tiene el sector frente al entorno macro de la economía del país, es necesario contemplar dos eslabones o ambientes transversales como lo son el organizacional y el institucional, para la integración de entidades reguladoras, entidades de investigación y normatividades y políticas sectoriales (Figura 1).

A partir de la caracterización de estos eslabones y la manera como se integran los flujos de material y capital a lo largo de la cadena, se tiene la base para identificar de qué manera se conjugan con la CF. Es importante notar que los proveedores de insumos se convierten en los actores en capacidad de ofrecer un paquete tecnológico en insumos, materiales, equipos entre otros para constituir de manera tangible la CF. 
Tabla 2. Caracterización de eslabones y segmentos de la cadena productiva de frutas y hortalizas en Colombia.

\begin{tabular}{|c|c|c|c|c|}
\hline Eslabón & Segmentos & Descripción & $\begin{array}{c}\text { Variable } \\
\text { primaria de } \\
\text { segmentación }\end{array}$ & $\begin{array}{c}\text { Otras } \\
\text { variable de } \\
\text { segmentación }\end{array}$ \\
\hline $\begin{array}{l}\text { Proveedor de Agro- } \\
\text { insumos }\end{array}$ & $\begin{array}{l}\text { Proveedor de Agroinsu- } \\
\text { mos Proveedores de } \\
\text { herramientas y maquinarias. } \\
\text { Proveedores de mate- } \\
\text { rial biológico, vegetal. } \\
\text { Proveedores de agroquímicos } \\
\text { e insumos de transformación. } \\
\text { Proveedor de insumos para } \\
\text { la industria de alimentos o } \\
\text { transformación } \\
\text { agroindustrial. }\end{array}$ & $\begin{array}{l}\text { Este primer eslabón de la cadena, agru- } \\
\text { pa a los integrantes que están relacio- } \\
\text { nados con el suministro de insumos, } \\
\text { material de siembra y otros productos } \\
\text { como maquinaria e insumos para agro- } \\
\text { industria, dirigidos para el eslabón de } \\
\text { productores. }\end{array}$ & $\begin{array}{l}\text { Tipo de insumo que } \\
\text { proveen }\end{array}$ & $\begin{array}{l}\text { Capacidad } \\
\text { logística. }\end{array}$ \\
\hline $\begin{array}{l}\text { Productor } \\
\text { primario }\end{array}$ & $\begin{array}{l}\text { Productores tecnificados } \\
\text { Productores no tecnificados }\end{array}$ & $\begin{array}{l}\text { Integra a los productores tecnifica- } \\
\text { dos y no tecnificados de productos } \\
\text { hortofrutícolas ubicados en las dife- } \\
\text { rentes regiones productoras del país }\end{array}$ & $\begin{array}{l}\text { Nivel tecnológico } \\
\text { de la unidad pro- } \\
\text { ductiva. }\end{array}$ & $\begin{array}{l}\text { Extensión de cul- } \\
\text { tivo. } \\
\text { Capacidad produc- } \\
\text { tiva }\end{array}$ \\
\hline $\begin{array}{l}\text { Comercializadores } \\
\text { de productos } \\
\text { hortofrutícolas en } \\
\text { fresco }\end{array}$ & $\begin{array}{l}\text { Comercializadores para } \\
\text { el mercado nacional } \\
\text { Comercializadores para el } \\
\text { mercado internacional. }\end{array}$ & $\begin{array}{l}\text { Integra a los actores encargados de } \\
\text { la comercialización de producto en } \\
\text { fresco o mínimamente procesado en } \\
\text { el mercado nacional e internacional. }\end{array}$ & $\begin{array}{l}\text { Segmento del mer- } \\
\text { cado atendido }\end{array}$ & $\begin{array}{l}\text { Cobertura } \\
\text { Capacidad } \\
\text { lógistica }\end{array}$ \\
\hline $\begin{array}{l}\text { Comercializadores } \\
\text { de productos } \\
\text { hortofrutícolas para } \\
\text { la agroindustria }\end{array}$ & $\begin{array}{l}\text { Acopiadores } \\
\text { Comercializador mayorista }\end{array}$ & $\begin{array}{l}\text { Este eslabón integra tanto a } \\
\text { acopiadores y mayoristas de las frutas y } \\
\text { las hortalizas, que vienen a ser el puen- } \\
\text { te entre el productor y la Agroindustria. }\end{array}$ & $\begin{array}{l}\text { Mecanismos de } \\
\text { interacción con los } \\
\text { eslabones } \\
\text { predecesores. }\end{array}$ & $\begin{array}{l}\text { Capacidad } \\
\text { lógistica }\end{array}$ \\
\hline $\begin{array}{l}\text { Agroindustria de } \\
\text { transformación }\end{array}$ & $\begin{array}{l}\text { Transformadores de Nivel I } \\
\text { Transformadores de Nivel II } \\
\text { Transformadores de Nivel III }\end{array}$ & $\begin{array}{l}\text { Este eslabón agrupa a todas aque- } \\
\text { llas empresas que tiene como ac- } \\
\text { tividad económica el procesa- } \\
\text { miento de las frutas y las hortalizas }\end{array}$ & $\begin{array}{l}\text { Grado de transfor- } \\
\text { mación al producto } \\
\text { en fresco. }\end{array}$ & $\begin{array}{l}\text { Demanda del } \\
\text { mercado. } \\
\text { Cantidad de } \\
\text { producción }\end{array}$ \\
\hline $\begin{array}{l}\text { Comercializadores } \\
\text { de productos de la } \\
\text { agroindustria }\end{array}$ & $\begin{array}{l}\text { Comercializador de } \\
\text { productos a nivel nacio- } \\
\text { nal (consumo interno). } \\
\text { Comercializador de produc- } \\
\text { tos a nivel internacional (ex- } \\
\text { portación). }\end{array}$ & $\begin{array}{l}\text { encargados de la comercializa- } \\
\text { ción, tanto a nivel nacional como } \\
\text { internacional. A nivel internacional } \\
\text { se encuentran los Traders y Brokers } \\
\text { internacionales, mientras que a ni- } \\
\text { vel nacional se comercializa con } \\
\text { grandes superficies comerciales, } \\
\text { hipermercados, supermercados y } \\
\text { empresas procesadoras nacionales. }\end{array}$ & $\begin{array}{l}\text { Mercado especiali- } \\
\text { zado }\end{array}$ & $\begin{array}{l}\text { Capacidad } \\
\text { lógistica }\end{array}$ \\
\hline Consumidor Final & $\begin{array}{l}\text { Consumidor final nacional } \\
\text { Consumidor final } \\
\text { internacional }\end{array}$ & 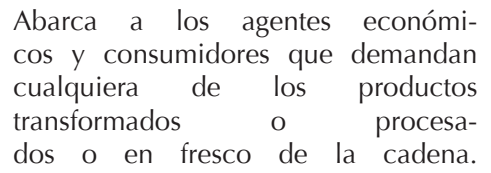 & $\begin{array}{l}\text { Segmento al que } \\
\text { pertenece }\end{array}$ & $\begin{array}{l}\text { Tipo de producto } \\
\text { que consume. }\end{array}$ \\
\hline
\end{tabular}

Fuente: Elaborado a partir de información encontrada en http://www.agrocadenas.gov.co, Agenda Prospectiva de investigación para la CP de Mango (Bonilla 2010), Agenda Prospectiva de Investigación para la Cadena de la uchuva (Bonilla, 2009). 


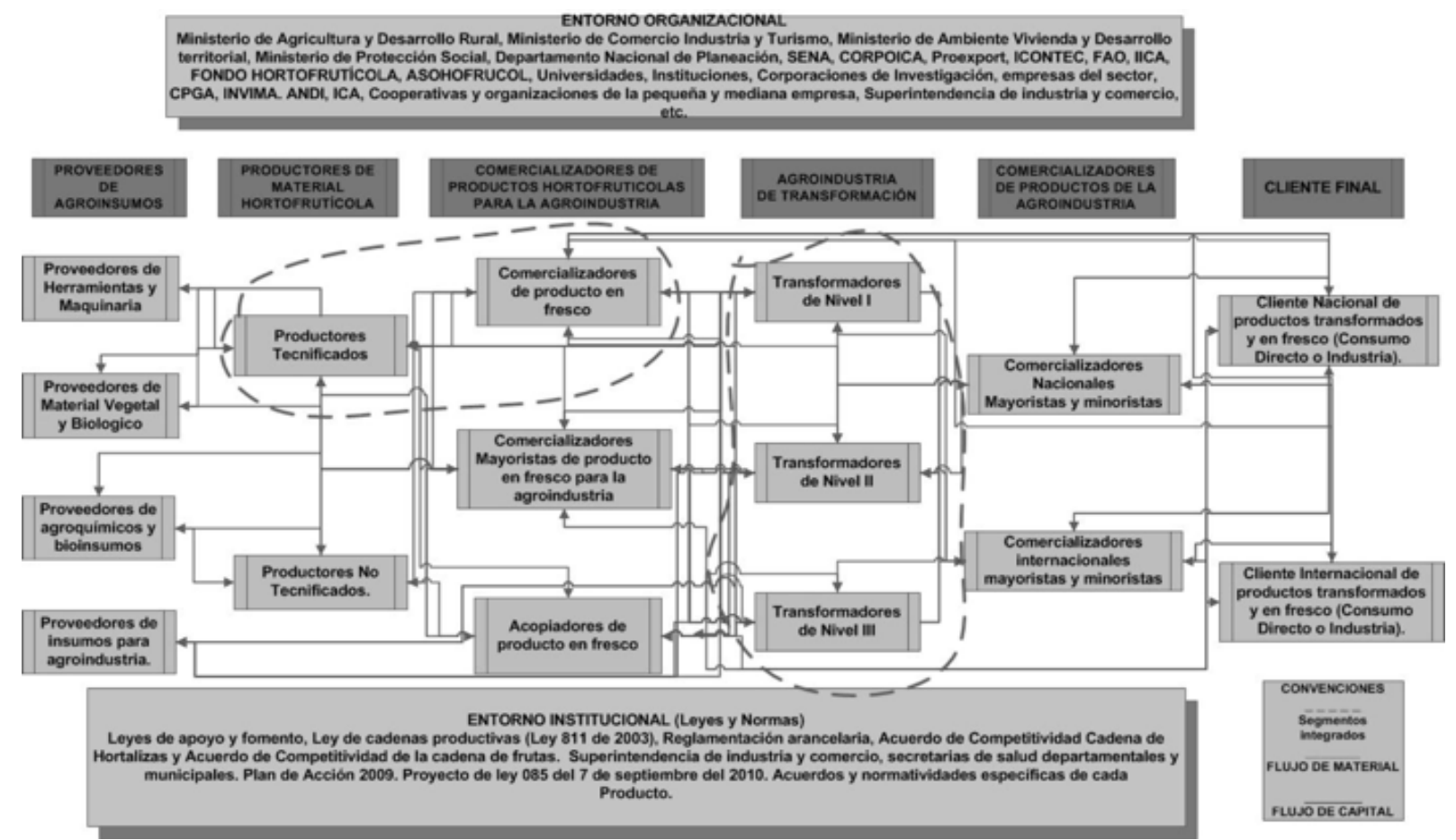

Figura 1. Modelo propuesto para la representación de la Cadena Productiva hortofrutícola. Fuente: Tomado de (Flórez, 2012)

Dentro del modelo establecido para la CP hortofrutícola, es necesario acotar los eslabones que competen al modelo de cadena de distribución específico para productos en fresco o mínimamente procesados, donde se conjugan las actividades de transporte de material, junto con las operaciones de manejo poscosecha de productos de la cadena constituyen el conjunto de procesos y operaciones a evaluar de manera conjunta frente a la CF.

\section{Etapa 2:}

La investigación a nivel mundial de la CF como factor especializado dentro de las cadenas de suministro, se ha desarrollado entorno a la integración de los eslabones a través de los cuales se presenta el flujo de materiales e información, con sistemas de trazabilidad que garanticen la temperatura en cada uno de los puntos críticos de control (PCC), en la tabla 3, se sintetizan los principales modelos de CF considerados para la CP hortofrutícola

Acotando la articulación de la CF como un proceso de transporte desde el punto de vista operativo, junto con lineamientos estratégicos de control desde la CP, el punto de confluencia es lograr un incremento en la calidad y cobertura del servicio frente a una disminución en los costos, sin embargo la dinámica actual del sector agrícola, enfoca la dinámica logística en un orden multivariado para pequeños lotes de transporte, donde el tiempo de vida útil del producto es directriz de estrategias en cuanto a priorización de órdenes, tiempos, asignación de entregas conjuntas entre otras, en busca de tener a tiempo, con calidad y al menor costo el producto en el eslabón del cliente final.

La integración de factores operativos y estratégicos debe soportarse bajo sistemas de información gerencial, que se convierta en punto de referencia frente a la identificación, seguimiento y control de puntos críticos en la distribución, por medio de la utilización de tecnologías blandas como software especializado para inventarios, ventas, datos de distribución, tiempos, generación de alertas tempranas para disminuir la perdida de producto. 
Tabla 3. Caracterización de modelos de CF afines para la CP hortofrutícola en

\begin{tabular}{cccccc}
\hline Autor & Descriptor & $\begin{array}{l}\text { Tecnologías } \\
\text { rramientas }\end{array}$ & $\begin{array}{c}\text { asociadas } \\
\text { de }\end{array}$ & $\begin{array}{c}\text { y } \\
\text { gestión. }\end{array}$ & $\begin{array}{l}\text { Elementos de interacción con las } \\
\text { cadenas }\end{array}$ \\
productivas.
\end{tabular}

Trazabilidad desde el material crudo o fresco, ( W a n g, Modelo de traza- seguimiento de código de barras. 2008) bilidad por uni- • HACCP para la resolución de indades productivas convenientes, como interrupción en el control de temperatura

Las operaciones de la CF deben ser los más cortas y rápidas posibles, lo que permitirá una reacción inmediata para reducir perdidas de material e información en cualquier eslabón.

Automatización posible de las activida- Sistemas de identificación de radio fre- des que componen el proceso logístico y (Montanari, Trazabilidad de la cuencia (RFID por sus siglas en inglés) distribución (seguimiento de productos en 2008) CF por sistemas de •Etiquetas (TAGS) que regis- tiempo real a lo largo de los eslabones). identificación de ra- tren la temperatura del producdio frecuencia - RFID to y la temperatura del entorno. Gestión del producto, la información, del (marcos estructurados) sistema tecnológico y de la calibración, abracando todos los factores de riesgo generados frente al manejo de la temperatura ( G u o j u n, Modelo Sistema de •Estándares de Medi- abarcando procesos de enfriamiento en: re2009) Seguridad para la CF das de Eficiencia para la CF. colección en el origen, enfriamiento previo, procesamiento, almacenamiento, empaque y transporte hasta el consumidor

( R a s p or,

2008); Modelo de integra- $\bullet$ HACCP

(Estrada-Flo- ción del consumidor • Buenas prácticas de alimentos diciones del entorno en tiempo real para su res, 2008) o cliente final a la CF. • Rastreo de posicionamiento geográfico trasmisión al cliente o al comercializador.r

(Yan \& Lee, Modelo integrativo de •Tecnologías RFID.

2009)

RFID para los eslabones •Etiquetas inteligentes, sensores de temde la cadena de distri- peratura, sistemas GPS, arquitectura del bución y productiva. sistema de monitoreo, diseño del software y del sistema de flujo de información.

Rastrear los productos de la CP a lo largo de la CF, en las etapas de procesamiento, almacenamiento, distribución y comercialización de la cadena de suministros las cuales tienen correspondencia con los eslabones de productores, transformadores, comercializadores y consumidores o cliente final, en tiempo real, donde el manejo de la temperatura como variable crítica de control.r

- Integración de tecnología RFID Acondicionar puntos de transporte logísti- Análisis de factores energéticos para co para cada eslabón en función de las vacadenas de suministro de productos riables del producto en cada una de las

(Kuo, 2010) Modelo de distri- perecederos, que contemplan la nece- etapas hasta llegar al consumidor final, gabución por nodos y sidad de tener un seguimiento de las rantizando control de temperatura, pérdiMultitemperaturas.. pérdidas exergéticas asociadas a los pro- das energéticas mínimas y calidad óptima. cesos productivos y de transformación

De los modelos analizados el modelo de Modelo de distribución por nodos y Multitemperaturas y el Modelo integrativo de RFID para los eslabones de la cadena de distribución y productiva, son los más relevantes para la integración.

\section{Etapa 3:}

A partir de la caracterización del modelo de integración óptimo para el sector hortofrutícola como lo es la CP de eslabones y segmentos, junto con la descripción de los modelos de distribución logística en CF pertinentes para la CPHF colombiana, así como de las necesidades puntuales de la misma, discriminadas en las estrategias necesarias para su implementación, es necesario identificar de qué manera se realiza la integración y como se desarrolla el seguimiento necesario para garantizar que la calidad del producto se mantenga en un margen acorde con la normatividad de este 
seguimiento y control a los factores TTT.

El monitoreo y control de la temperatura a lo largo del proceso de distribución logístico en la CF hortofrutícola, se relaciona con los intereses particulares en campos como la economía, la innovación, la microbiología, principalmente enfocados en satisfacer los requerimientos del cliente final. A partir del modelo de CP establecido se realiza la identificación de los puntos críticos de control (PPC) en cada uno de los eslabones, como se consigna en la tabla 4.

Desde el punto de vista de las tecnologías duras estructurando los factores tecnológicos y no tecnológicos que implican la consolidación de la $\mathrm{CF}$, dentro de la $\mathrm{CP}$, se pueden definir eslabones para este modelo de distribución adaptativo. Los eslabones que forman esta cadena serían los siguientes: almacenes frigoríficos situados en las zonas productoras; vehículos de transporte frigoríficos; almacenes frigoríficos generales, comerciales y de consumo; transportes frigoríficos urbanos; cámaras y muebles frigoríficos de establecimientos públicos o institucionales, de supermercados y de los detallistas y, el frigorífico doméstico, así como las tecnologías de monitoreo y control de la temperatura y humedad.

Canella (2010), propone que el análisis de los cuatro arquetipos se realice bajo el esquema propuesto del depósito del agua. Los cuatro arquetipos a analizar para la $\mathrm{CP}$, son a saber: Cadena de suministro tradicional, a información compartida, con pedido gestionado por el proveedor y cadena sincronizada (Tabla 5).

Para la CP hortofrutícola nacional, el líquido, serán los productos frescos, mínimamente procesados y procesados, los cuales transitan por la cadena, las válvulas o reglas de pedido son las decisiones que se deben tomar para regular los productos en este caso sujetas a la temperatura y el tiempo de entrega de los mismos, acorde con la capacidad de manejo y las cisternas, son el inventario y las condiciones de almacenamiento optimo del mismo.

Los cuatro arquetipos descritos con antelación se presentan en cualquier actividad de logística y son todos extensivos a la CP, sin embargo, sin embargo, el arquetipo ideal teniendo en cuenta las particularidades de esta ya que permite hacer seguimiento a tiempos y temperaturas de entrega.

Pese a que los cuatro arquetipos pueden ser aplicados a cadenas puntuales de ciertos productos, teniendo en cuenta que este arquetipo ideal no contempla limitaciones como la localización geográfica de los miembros de la $\mathrm{CP}$, el tipo de demanda entendida para productos hortofrutícolas como la estacionalidad de la misma, características del producto donde hay una heterogeneidad total entre la productos en fresco, mínimamente procesados y procesados, costos del sistema de trazabilidad RFID y TIC, cambio organizacional en la consolidación de agronegocios sostenibles y grado de apertura de la información considerando esta como una ventaja competitiva. En la figura 2 , finalmente se tiene que los eslabones de análisis de la CP frente a la CF son los mismos que se contemplan en la cadena logística.

En este sentido el modelo de integración de la CP y la CF, se estructura de manera horizontal entre los segmentos de los eslabones de productores, comercializadores de productos para la agroindustria, agroindustria de transformación, comercializadores deproductos de la agroindustria y cliente final, se manejan bloques de integración para estos 3 eslabones, donde el eslabón de comercializadores integra los segmentos correspondientes a la CP de comercializadores y transformadores. La numeración de 1 a 4, tipifica las actividades de la CF que se contemplan en los segmentos integrados, para almacenamiento, manipulación, comercialización. El flujo de material e información va de izquierda 
Tabla 4. Puntos Críticos de Control identificados para los eslabones de la Cadena Productiva hortofrutícola en Colombia.

Eslabón

Definición del eslabón
PPC

Actividades y etapas que comprenden El punto crítico de control de este eslabón, son la conformación de producto, desde la los manejos o buenas prácticas agrícolas junto perspectiva agroindustrial, determina la con los criterios de selección frente al producto a calidad inicial del producto que ingresa comercializar, los cuales acorde con la

PRODUCTORES PRIMARIOS a la cadena de suministro al igual que a normatividad colombiana, se sugiere o deben estar la cadena de frio, las cuales comprenden sujetos a las disposiciones de la NTC respectiva, desde la siembra, sostenimiento, así como los criterios establecidos por el nicho de fertilización, mantenimiento, reco- mercado al cual va dirigido, junto con los de los lección, clasificación y selección actores encargados de la distribución. Los comercializadores de productos Dado que no se manejan ningún tipo de aditivos, COMERCIALIZADORES agrícolas en fresco de la agroindus- ni conservantes, solo se cuenta con la tecnoloCOMERCIALIZADORES tria, son aquellos que constituyen el gía para conservar la temperatura de preservación DE PRODUCTOS EN canal directo entre el producto recién en un tiempo determinado, el punto crítico de FRESCO O MÍNIMA- clasificado y seleccionado y el cliente final control para este eslabón, es la temperatura de MENTE PROCESADOS DE LA AGROINDUSTRIA refrigeración o congelación la cual depende del sitio de destino del producto de consumo, así como la manipulación en la salida y entrega del mismo

Gran parte de los productos agrícolas en Nuevamente el punto crítico de fresco provenientes del eslabón de control es el manejo de temperatura

COMERCIALIZADORES DE PRODUCTOS AGRÍCOLAS PARA LA AGROINDUSTRIA. productores primarios, se destinan para durante el transporte a las unidades productivas, el la agroindustria de transformación, almacenamiento en las mismas, y los posibles donde se efectúan procesos de valor agre- choques térmicos durante la manipulación gado en cuanto a transformación del del producto insumo recibido, obteniendo productos que van desde jugos, pulpas, mermeladas, hasta yogures, cereales, entre otros.

Actividades y etapas que comprenden El punto crítico de control de este eslabón, son la conformación de producto, desde la los manejos o buenas prácticas agrícolas junto perspectiva agroindustrial, determina la con los criterios de selección frente al producto a calidad inicial del producto que ingresa comercializar, los cuales acorde con la

PRODUCTORES PRIMARIOS a la cadena de suministro al igual que a normatividad colombiana, se sugiere o deben estar la cadena de frio, las cuales comprenden sujetos a las disposiciones de la NTC respectiva, desde la siembra, sostenimiento, así como los criterios establecidos por el nicho de fertilización, mantenimiento, reco- mercado al cual va dirigido, junto con los de los lección, clasificación y selección actores encargados de la distribución.

Eslabón con el más alto grado de desarrollo El factor crítico de control es la calidad del producto tecnológico, acorde con la exigencia de los en fresco recibido por el eslabón de comercializa-

AGROINDUSTRIA TRANSFORMACIÓN
DE productos esperados por los mercados obje- dores para la agroindustria y los controles de tempetivos de cada aparato productivo, así como ratura realizados hasta la introducción del mismo al los criterios de calidad del consumidor proceso productivo, sin embargo es importante contrarrestar la información recibida antes del proceso de transformación a través de pruebas de trazabilidad a nivel laboratorio, que garanticen la calidad del lote.

COMERCIALIZA DORES Eslabón con el más alto grado de desarro- Enestepuntosearticulan, lasBPM,BPD,HACCPylosfacDE PRODUCTOS DE LA llo tecnológico, acorde con la exigencia de tores tecnológicos necesarios para garantizar calidad. AGROINDUSTRIA los productos esperados por los mercados objetivos de cada aparato productivo, así como los criterios de calidad del consumidor

El cliente final último eslabón de la cade- El punto crítico de control es el manena productiva, orientador de las prácti- jo del producto desde la salida de la unicas de calidad a través de toda la cade- dad de comercialización hasta llegar al hogar.

CLIENTE FINAL na, es el encargado de valorar desde la perspectiva del consumo el valor del producto, donde existe el riesgo de un mal manejo por parte del mismo que afecte de manera subjetivalapercepción real del mismo 
Tabla 5. Análisis de los cuatro arquetipos de Cadenas de suministros frente a los eslabones base de la cadena productiva.

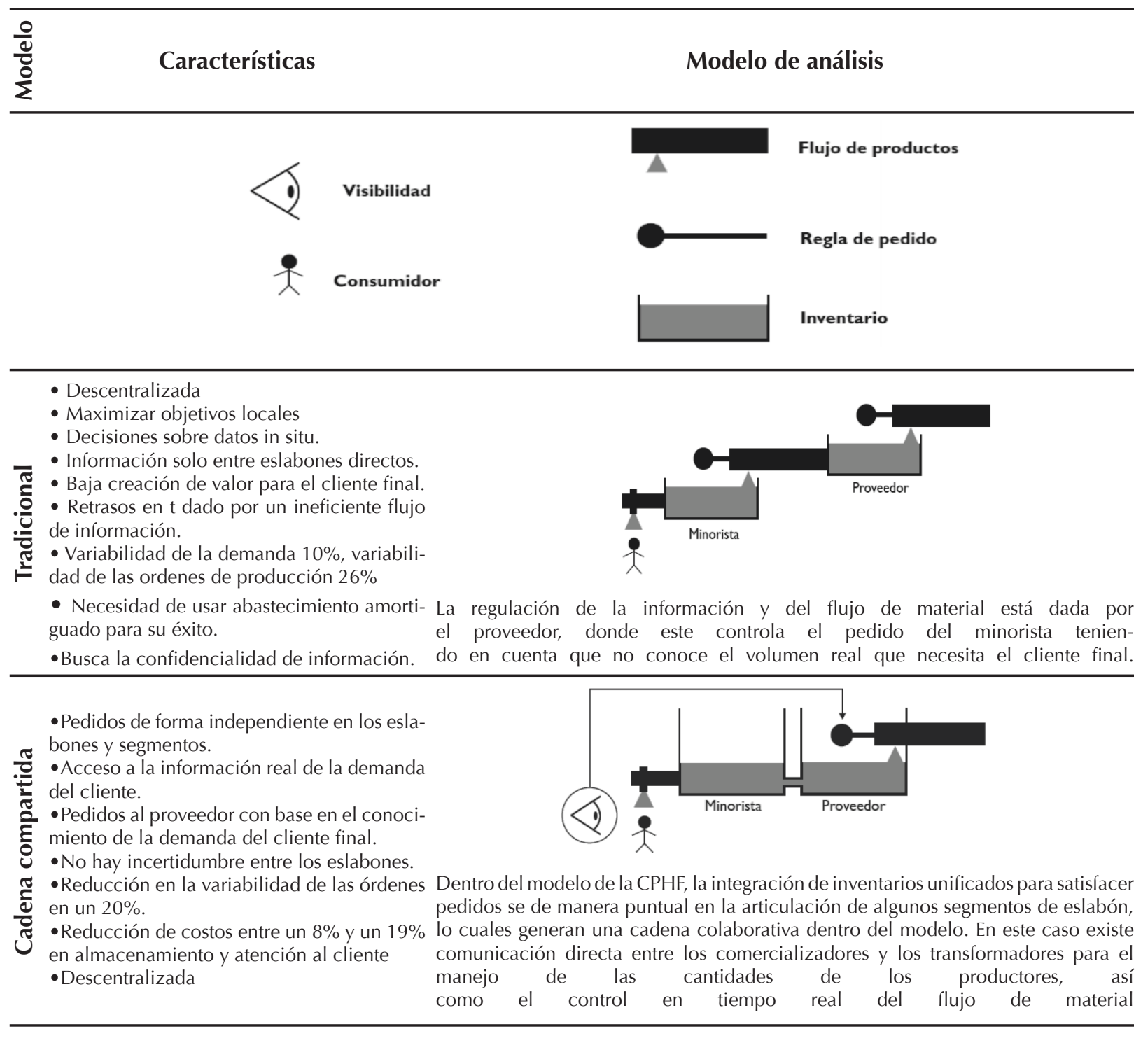

- Estructura centralizada

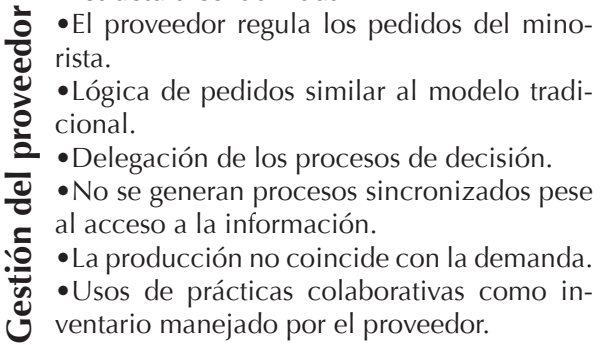

¿ El proveedor regula los pedidos del mino을 cional.

¿ Delegación de los procesos de decisión.

¿ No se generan procesos sincronizados pese al acceso a la información.

Usos de prácticas colaboravas como inventario manejado por el proveedor.

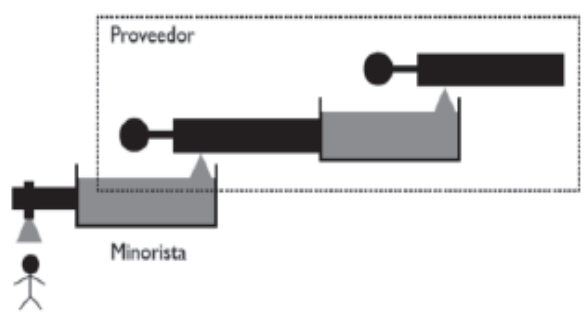

La integración de eslabones de manera horizontal, se presenta en los segmentos, donde algunos productores se articulan con transformadores y/o comercializadores para tener un inventario unificado en términos de la demanda del cliente final, esto limita la heterogeneidad de la calidad del producto, pero disminuye la cobertura de actores dado que no todos manejan niveles tecnológicos similares. 
- Modelo centralizado

- Pedidos en tiempos homólogos.

- Logística en tiempo real

- Información sincronizada de inventarios,

producto en movimiento y ventas.

- Ordenes de producción en función de la demanda.

-Todos los inventarios de la cadena se consi-

deran un único inventario

-Regulación de todos los flujos de material

e información

-Disminución del efecto látigo

-Disminución de costos en un 50\%

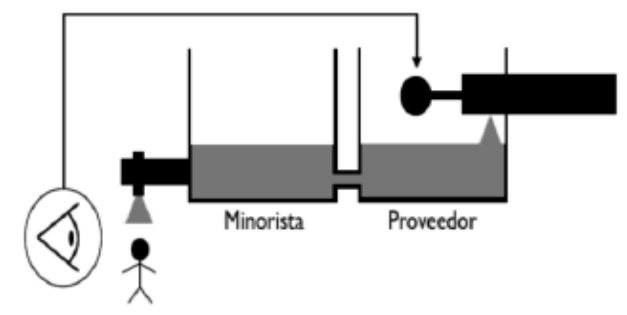

La integración de inventarios unificados para satisfacer pedidos se de manera puntual en la articulación de algunos segmentos de eslabón, lo cuales generan una cadena colaborativa dentro del modelo. En este caso existe comunicación directa entre los comercializadores y los transformadores para el manejo de las cantidades de los productores, así como el control en tiempo real del flujo de material a derecha y se tiene de derecha a izquierda retroalimentación de información. En este punto se identifican cuatro puntos críticos de control como máximo en el modelo de la integral de la CP hortofrutícola y la cadena de frío HF, sujetos de ser gestionados a través del análisis HACCP*. Teniendo en cuenta el modelo establecido en la figura 2, los PCC a controlar son:

- Productores (PCC interno): durante la manipulación inicial después de las etapas de cosecha, manejo post cosecha de producto en fresco, control de las propiedades fisicoquímicas, madurez, vida útil, empaques y embalaje desde la unidad productiva hasta almacenamiento inicial o transporte según se articule la CP de eslabones y segmentos.

- Productores (PCC externo): condiciones de almacenamiento, frente al transporte del producto en fresco al eslabón integrado de comercializadores o de manera directa al cliente final si este requiere producto en fresco.

\section{- Comercializadores (PCC interno, segmento} de comercializadores en fresco): la manipulación del producto en fresco por parte de comercializadores debe cumplir con un control de la trazabilidad del producto recibido frente a los requisitos del cliente final, o de los eslabones integrados subsecuentes, tecnología como RFID puede ser integrada desde este punto de la cadena.

- Comercializadores (PCC interno, segmento de transformadores): teniendo en cuenta que en este eslabón se incrementa el valor agregado dependiendo del grado de transformación del producto en fresco, la especificidad del manejo de la temperatura en manipulación y almacenamiento depende de los procesos a los que se somete el producto. RFID dentro de las unidades de refrigeración es recomendable

- Comercializadores
comercializadores de productos de la
agroindustria): la etapa de distribución de
productos transformados debe ser efectuada
en trasporte especializado acorde con las
condiciones de almacenamiento requeridas
para cada producto en las unidades de
comercialización. Tecnología RFID requerida

- Comercializadores (PCC externo, segmento de comercializadores en fresco): este primer punto de control externo depende del tiempo

La metodología HACCP es un análisis sistemático de cada una de las etapas involucradas en un proceso para establecer situaciones de riesgo que puedan alterar la inocuidad del alimento, para establecer medidas de control y está diseñado para minimizar el riesgo. Para el caso puntual de la cadena hortofrutícola integrada a la cadena de frío, es el control del incremento o deceso intempestivo de la temperatura ocasionando perdida de producto y la calidad del mismo. El análisis HACCP estándar sigue siete pasos básicos: (1) Análisis preventivo (2) Identificación de PCC, (3) Determinación de límites críticos, (4) Monitoreo de cada PCC, (5) Aplicación de acciones correctivas a efectuar cuando ocurra una desviación del límite crítico, (6) Desarrollo e implementación de un sistema de mantenimiento de registros y (7) Procedimientos de verificación. 


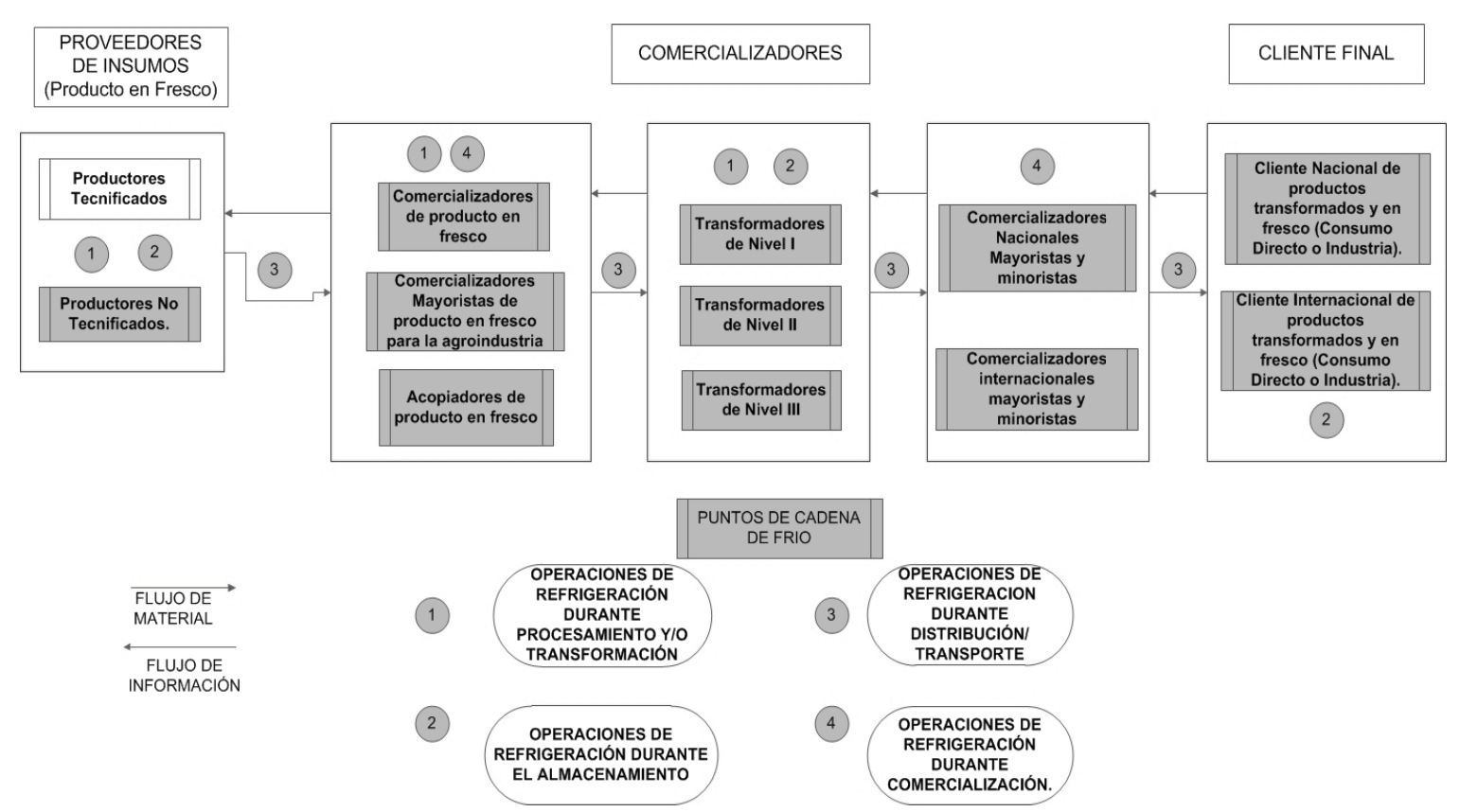

Figura 2. Modelo integrado de la Cadena de frío a la cadena productiva hortofrutícola.

Fuente. Autor

de transporte desde la comercialización al eslabón integrado de destino, en términos de las condiciones iniciales de refrigeración o congelamiento y las condiciones finales, para tener en cuenta el tiempo. Manipulación donde se rompe la CF entre la salida del transporte y la llegada al destino final. Esto depende de la veracidad de los datos entregados de izquierda a derecha en la cadena, se recomienda tecnologías RFID.

\section{- Comercializadores (PCC externo,} segmento de transformadores): de manera homologa al análisis anterior, solo cambia la tipificación del producto en seguimiento en la fase de almacenamiento y transporte. - Comercializadores (PCC externo, comercializadores de productos de la agroindustria) homologa al análisis anterior, solo cambia la tipificación del producto en seguimiento en la fase de almacenamiento. La fase de transporte solo se contempla para el cliente final internacional.

- En cuanto al cliente final, la CF tiene en este punto su mayor déficit en cuanto a PCC teniendo en cuenta que la manipulación del mismo depende del mismo, del propósito de uso y de las instalaciones con que se cuenta, en particular para el cliente final nacional.

Como se ha evidenciado a través del análisis y construcción del modelo de la $\mathrm{CP}$, el modelo de la CF, el modelo de la CdS, para finalmente llegar al modelo integrativo de la CF y la CP, en específico para el sector hortofrutícola, la variable de seguimiento en cuanto a trazabilidad de material, información, calidad y control por HACCP para los PCC es la temperatura, la cual incide directamente en el producto en términos de proceso, transporte, almacenamiento, viéndose reflejada en términos organizacionales en costos, productividad y competitividad.

El fortalecimiento de un sector con niveles de heterogeneidad a lo largo de toda la cadena de valor, de cualquier aparato productivo como lo es la CP hortofrutícola, presupone la necesidad de identificar y caracterizar los niveles de interacción frente a un objetivo o visión compartida que es entregar al cliente final un producto 
inocuo, organolépticamente diferenciado y en las cantidades y calidades requeridas así

El análisis de las interacciones de eslabones y segmentos que pueden ser tipificadas para cualquier producto hortofrutícola desde su presentación en fresco hasta productos con valor agregado como pulpa, néctares, jugos y yogures, permite proponer esquemas de asociatividad impulsados por lineamiento de política pública para cadenas productivas, logrando acceder a recursos de financiación para el desarrollo tecnológico con mayor facilidad dentro de las áreas prioritarias establecidas en los acuerdos de competitividad del sector y en los esquemas de los TLC.

La temperatura, la humedad y el tiempo son las variables claves de control de los productos hortofrutícolas, estas pueden ser controladas, monitoreadas y modificadas a través de la CF como herramienta de integración tecnológica en proceso y producto directamente ligada a las actividades de almacenamiento y transporte propias de los modelos de distribución logística e inventarios que hacen parte de la cadena de suministros como parte integral de la CP, lo que le permite a estas trascender de una vocación de comercio interno a un enfoque de exportación.

El análisis de los modelos de CF adaptados para la comercialización de productos hortofrutícolas a nivel mundial, el grado de pertinencia frente a la trazabilidad de las variables propuestas a través del HACCP y los PCC como método de seguimiento e identificación, permitió identificar los modelos logísticos, de distribución, que contemplan tanto almacenamiento como transporte refrigerado a lo largo de los diferentes actores de la cadena de suministro, para garantizar la conformación de CF en términos de procesos y procedimientos.

Por otra parte, teniendo en cuenta que parte del grado de innovación de la metodología de cadenas productivas, son las integraciones horizontales y verticales de segmentos de eslabón para la conformación de clústeres que optimicen los flujos de material e información, el arquetipo óptimo para esta característica de las cadenas es el de cadena sincronizada el cual es coherente con el HACCP como herramienta de anclaje del modelo de CP con los modelos de CF propuestos

La consolidación del modelo de CP, la identificación del arquetipo de cadena sincronizada, óptimo para poder adaptar los modelos de cadena de frío identificados para el manejo de alimentos y productos con grado de perecibilidad por temperatura, a través de PCC permite la articulación necesaria con la cual se formula el modelo de integración de la CP hortofrutícola con la CF (Figura 2), como un estándar sobre el cual a través de PCC se pueden establecer los insumos tecnológicos y no tecnológicos para optimizar la cadena de distribución.

Finalmente la cadena de frío como alternativa para el fortalecimiento del sector agroindustrial colombiano frente a la demanda internacional y nacional de volúmenes cada vez mayores de productos agroindustriales con criterios de calidad rigurosos, no debe limitarse al análisis y pertinencia de los modelos o no que puedan ser integrados, a las cadenas productivas nacionales como mecanismos de impulso tecnológico, sino deben ser sujetos de evaluación, caracterización e implementación en unidades reales, desde el punto de vista de la transferencia y adaptación de tecnologías pertinentes que garanticen de manera global desde la cadena de suministro como establece (Herrera, 2008): "...armonizar todas las actividades asociadas con el flujo y transformación de bienes desde la etapa de las materias primas hasta el usuario final en los dos sentidos, desde arriba y hacia abajo dentro de la cadena de abastecimientos...", con el objetivo de garantizar incremento en los indicadores de competitividad y productividad a nivel sectorial, de cadena y empresa. 


\section{CONCLUSIONES}

El modelo de integración CP-CF propuesto retoma de manera sistémica aquellos esquemas desarrollados con antelación, reflejando los aportes de cada uno y solventando los aspectos no contemplados, dentro de la metodología de análisis, se convierte en el mapa de estudio sobre el cual se deben implementar las mejoras identificadas en los diagnósticos y caracterizaciones del sector.

La temperatura, la humedad y el tiempo son las variables claves de control de los productos hortofrutícolas, estas pueden ser controladas, monitoreadas y modificadas a través de la cadena de frío como herramienta de integración tecnológica en proceso y producto.

Es necesario queseelabore un modelo adaptativo entre la cadena de frío, la cadena productiva y la cadena de suministros, que contemple no solo el factor tecnológico, sino también los factores de costos, factores de inventarios, redes de distribución, tipos de vehículos, costos logísticos detallados, metodologías de seguimiento y trazabilidad a productos.

Dentro de los aspectos relevantes a nivel técnico, identificados durante el análisis de la cadena hortofrutícola, se tiene que dentro del manejo de la cadena de frío, es necesario considerar dentro de estudios de la oferta tecnológica tanto nacional como internacional en términos de unidades de refrigeración móviles (Transporte Refrigerado), unidades de refrigeración estáticas (Cuartos y cabinas con acondicionamiento de aire frío), tecnología de empaques funcionales, entre otras, óptimas para cada eslabón de la cadena y preferiblemente para cada segmento, en atención a la diversidad de productos que se pueden manejar por niveles de transformación como factor de valor agregado y ventaja competitiva.

\section{REFERENCIAS}

Asohofrucol y MADR. 2011. Plan frutícola y hortícola nacional. Bogotá D.C: Ministerio de Agricultura y Desarrollo Rural.

Beilin, L. 2004. Food maintains freshness with the cold chain. Chemical industry publishing house.

Bonilla, M., Arias, P., Landínez, L., Moreno, J., Cardozo, F., y Suárez, M. 2009. Agenda Prospectiva de Investigación y Desarrollo Tecnológico para la cadena productiva de la Uchuva en Fresco para Exportación en Colombia. Bogotá D.C: Giro Editores Ltda.

Bonilla, M., Arias, P., Chica, J., Mejía, A., y Landínez, L. 2010. Agenda Prospectiva de Investigación y DesarrolloTecnológico para la Cadena Productiva de mango criollo procesado para exportación en Colombia. Bogotá D.C: Giro Editores Ltda.

Cannella, S. 2010. Los Cuatro Arquetipos de la Cadena de Suministro. Universia Business Review, 134-149.

Castellanos, O., Torres, L. y Flórez, D. 2010. Agenda Prospectiva de Investigación y Desarrollo para la Cadena Productiva de la Panela y su agroindustria. Bogotá D.C: Giro Editores Ltda.

Castro, A. 2006. Taller de Prospectiva en cadenas productivas. Taller de Prospectiva en cadenas productivas. Bogotá D.C: IICA- Instituto interamericano de cooperación agrícola.

Castro, A. y Lima, S. 2001. Análisis prospectivo de cadenas productivas agropecuárias. Lima: EMBRAPA.

Castro, A., Lima, S., Goedert, W., Freitas, A., y 'Vasconcelos, J'. 1998. Cadeias productivas e Sistemas Naturais Prospeccao Tecnológica. Brasilia: Embrapa.

Cruz, R. 2009. Effect of cold chain temperature abuses on the quality of frozen watercress (Nasturtium officinale $\mathrm{R}$. Br.). Journal of Food Engineering, 90-97. https://doi. org/10.1016/j.jfoodeng.2009.03.006 
Cuevas, R. 2005. Diagnóstico Prospectivo de la cadena de leche de vaca en el estado de Hidalgo. Hidalgo, México: Informe técnico sin publicar INIFAP y Fundación Hidalgo Produce A.C.

Estrada, S. 2008. RFID Technologies for Cold Chain Applications. Intergovernmental organization for the development of refrigeration bulletin 4, 4-8.

Fischer, C., Gonzalez, M. Henchion, M., y Leat, P. 2007. Trust and economic relationships in selected European agrifood chains. Acta Agriculturae ScandSection C, 4(1), 40-48. https://doi. org/10.1080/16507540701192543

Fischer, C., Hartmann, M., Reynolds, N., Leat, P., Revoredo-Giha, C., Henchion, M., y Gracia, A. 2008. Agrifood chain relationships in EuropeEmpirical evidence and implications for sector competitveness. In 12th Congress of the European Association of Agricultural Economists (EAAE), Mathijs E., Verbeke W., de Frahan $\mathrm{BH}$ (edts.), Ghent, Belgium.

Flórez, D. 2012. Diseño de una metodología de análisis exergético de la cadena de frío en cadenas productivas hortofrutícolas para evaluar la productividad y competitividad: caso de estudio mora de castilla. Bogotá D.C: Universidad Nacional de Colombia. Tesis de Maestría

Flórez, D y Cifuentes, H. 2013. An Exergetic Cold Chain Methodological Analysis on Horticultural Productive Chains to Evaluate Productivity and Competitiveness: Study Case Andean Blackberry. International Journal of Agriculture and Forestry, 16-28.

Friend, A. 2001. Cold Chain for Agricultural Products in Rwanda. Kigali, Rwanda: Agricultural Analyses and Design. United States Agency for International Development. Chemonics international, inc.

Fuller, R. 1998. Cold Store: Equipment and Maintenance. En A Practical Guide to the Cold Chain From Factory to Consumer pag 5-15
García, M., y Poole, N. 2004. The development of private fresh produce safety standards: implications for developing Mediterranean exporting countries. Food Policy, 229-255. https://doi. org/10.1016/j.foodpol.2004.04.002

García, J. 2009. Estrategias de cooperación delos productores defrutasy hortalizas. Una cooperación trasatlántica. CIRIEC-Revista económica, 193-216.

Guojun, J. 2009. Research on the Security of Cold-Chain Logistics. IEEE, 757-761. https://doi. org/10.1109/ICSSSM.2009.5174982

Herrera, O. 2008. Análisis cualitativo desde la perspectiva de la gestión del conocimiento en la cadena de abastecimiento de alimentos. Revista Ciencias Estratégicas, 265-279.

Jol, S. 2007. The Cold Chain, one link in Canada's food safety initiatives. Food Control, 713-725. https://doi. org/10.1016/j.foodcont.2006.03.006

Kuo, J. 2010. Developing an advanced Multi-Temperature Joint Distribution System for the food cold chain. Food Control, 559-566. https://doi. org/10.1016/j.foodcont.2009.08.007

Likar, K. 2006. Cold chain maintaining in food trade. Food Control, 108$113 . \quad$ https://doi.org/10.1016/j. f o o d c o n t.2004 .0 9.009

MADR, 2016. Siembra. Obtenido de Sistema de información de innovación agropecuaria: h t tp://www.siembra.gov.co/

Montanari, R. 2008. Cold Chain Tracking: A Managerial Perspective. Trends in Food Science\&Technology, 425-431.https:// doi.org/10.1016/j.tifs.2008.03.009

Raspor, P. 2008. Total food chain safety: How good practices can contribute? Trends in Food Science Technology, 405-412. https://doi. org/10.1016/j.tifs.2007.08.009 
Rediers, H. 2009. Evaluation of thecoldchain of fresh-cut endive from farmer to plate. Postharvest Biology and Technology, 257-262. https://doi.org/10.1016/j. postharvbio.2008.07.017

Rodríguez, V., Amorro, I., y Álvarez, f. 2011. Setting parameters in the cold chain. Tecnura, 71-81.

Torres, J. 2006. Estrategias de control de la cadena de frío y su impacto en la calidad y seguridad de los alimentos: Productos Hortofrutícolas en el desarrollo agroalimentario". Ibagué.

Viteri,M.2003. Logísticaenlacadenadefrutas y hortalizas frescas. IDIA XXI Revista de Información sobre Investigación y Desarrollo Agropecuario, 176-180.
Wang, L. 2008. A Research on Related Questions of Chinese Food Cold Chain Development," in. International Conference on Management of e-Commerce and e-Government, págs. 18-21.

Yan, B., y Lee, D. 2009. Application of RFID in Cold Chain temperature Monitoring System. ISECS International Colloquium on Computing, Communication, Control, and Management, págs. 258-261. https://doi. org/10.1109/CCCM.2009.5270408 